CLINICAL STUDY

\title{
Regular aerobic exercise training improves endothelium- dependent arterial dilation in patients with subclinical hypothyroidism
}

\author{
Guang-da Xiang, Jinhui Pu, Huiling Sun, Linshuang Zhao, Ling Yue and Jie Hou \\ Department of Endocrinology, Wuhan General Hospital of Guangzhou Command, Wuluo Road 627, Wuhan 430070, Hubei Province, \\ People's Republic of China
}

(Correspondence should be addressed to GD Xiang; Email: guangda64@hotmail.com)

\begin{abstract}
Objective: Impairment of flow-mediated endothelium-dependent arterial dilation (FMD) exists in patients with subclinical hypothyroidism (sHT). Several studies showed that exercise training can improve FMD in patients with type 1 and type 2 diabetes. Therefore, we hypothesized that exercise training can also improve FMD in subjects with sHT. The purpose of the study was to test this hypothesis.

Research design and methods: We selected 30 sedentary women with sHT and 27 sedentary healthy women with euthyroid. All individuals participated in an exercise training of 6 months. Before and after exercise training, high resolution ultrasound was used to measure FMD.

Results: At baseline, FMD among subjects with sHT was $3.87 \%$, which was significantly lower than that in controls $(5.98 \% ; P<0.001)$. After 6 months of exercise, there was a remarkable increase in FMD $(31.3 \%)$ and $\mathrm{VO}_{2} \max (36.7 \% ; P<0.01)$, and significant decreases in total cholesterol $(20 \%)$, low-density lipoprotein cholesterol (LDL; 29\%), triglycerides (TG; 47.6\%), and C-reactive protein (CRP; $61.5 \% ; P<0.05)$ were observed over the exercise in patients with sHT. The absolute changes in FMD showed significant correlation with changes in LDL $(r=-0.596)$, TG $(r=-0.532)$, and CRP $(r=-0.511 ; P<0.01)$, and multiple regression analysis showed changes of LDL, TG, CRP were significant determinants of changes of FMD in sHT patients during exercise course.

Conclusion: Regular aerobic exercise improves FMD in sHT patients, and changes of lipids and inflammation during the exercise period may partially contribute to the improvement of endothelial function.
\end{abstract}

European Journal of Endocrinology 161 755-761

\section{Introduction}

The endothelium plays an important role in modulating vascular function and structure through the production of vasodilator and vasoconstrictor substances (1). Endothelial dysfunction reflects the disordered physiology of several endothelium-derived vasoactive factors, in particular nitric oxide (NO) (2), and is an early physiological event in atherosclerosis (1). Patients with subclinical hypothyroidism (sHT), a disorder characterized by elevated serum TSH levels despite normal free hormone (free tri-iodothyronine $\left(\mathrm{fT}_{3}\right)$ and free thyroxine $\left.\left(\mathrm{fT}_{4}\right)\right)$ values, are associated with increased prevalence of atherosclerotic lesions and cardiovascular events $(3,4)$. Recently, many studies showed that impairment of flow-mediated endothelium-dependent arterial dilation (FMD) exists in patients with sHT $(5,6)$. Many studies suggested that treatment of sHT can improve cardiovascular health $(5,7)$. The crucial mechanism of this endothelial dysfunction remains unclear.
Exercise training can improve vascular endothelial function in patients with prediabetes (8), type 1 diabetes (9) and type 2 diabetes (10) as well as in the nondiabetic population (11). To date, no studies on endothelial function in subjects with sHT in response to exercise training have been reported. Therefore, we hypothesized that exercise training can also improve FMD in subjects with sHT. The purpose of the study was to test this hypothesis.

\section{Subjects and methods}

\section{Subjects}

From January 2002 to January 2006, 30 sedentary Chinese Han women with sHT (physical exercise less than once a week), referred to our hospital for healthy examination, between 46 and 65 years of age, mean age $53 \pm 8$ years, were studied. All patients with sHT were newly diagnosed, as were those with Hashimoto's 
thyroiditis, who were positive for both antithyroid peroxidase (TPO-Ab) and antithyroglobulin ( $\mathrm{Tg}-\mathrm{Ab}$ ) antibodies. The diagnosis of sHT was established on the basis of the elevated TSH levels, and normal $\mathrm{fT}_{3}$ and $\mathrm{fT}_{4}$ values. During the same period, 27 sedentary healthy women who were euthyroid (physical exercise less than once a week, from the medical staff in our hospital), aged 46-63 years, mean age $52 \pm 9$ years, were selected as control. All individuals participated in an exercise training program of 6 months. Of them, two patients and two healthy women discontinued the study after 1 month of training because of time constraints. All subjects including sHT and controls were postmenopausal women. Obese subjects, (body mass index (BMI) $>30 \mathrm{~kg} / \mathrm{m}^{2}$ ) smokers, and those with hypertension, clinical detectable coronary artery disease, and other diseases were excluded from the study. Also, no patient was taking any drugs, such as estrogen supplements, $\mathrm{T}_{4}$, diuretics, antihypertensive and hypolipidemic drugs. All subjects gave informed consent. The study protocol was in agreement with the guidelines of the local ethics committee, and approved by the ethics committee at our hospital.

\section{Methods}

Laboratory methods In patients with sHT, vascular and laboratory examinations were performed 1-3 days before and 6 months after the initiation of the exercise training program. After a $12-\mathrm{h}$ fasting period, blood was obtained for measuring plasma lipids, glucose, thyroid function, and other parameters. Serum total cholesterol (TC), low-density lipoprotein cholesterol (LDL), triglycerides (TG), and high-density lipoprotein cholesterol (HDL) were measured enzymatically. Apolipoprotein A1 $\left(\mathrm{ApoA}_{1}\right)$ and $\mathrm{ApoB}$ were measured by immunoturbidimetry. Serum lipoprotein (a) (Lp(a)) levels were measured by an ELISA method. Fast blood glucose (FBG) was measured by a glucose oxidase procedure. $\mathrm{C}$-reactive protein (CRP) concentrations were measured using the CRP (Latex) ultrasensitive assay. The concentrations of $\mathrm{fT}_{3}$ (normal range, 3.20-9.20 pmol/l) and $\mathrm{fT}_{4}$ (normal range, $9.10-25.60 \mathrm{pmol} / \mathrm{l}$ ) were measured by RIA, and TSH (normal range, 0.3-5.5 mU/l) was determined with an ultrasensitive IRMA method. Tg-Ab was measured by specific IRMA (normal range, $<50 \mu / \mathrm{ml}$ ); TPO-Ab was measured by specific RIA (normal range, $<10 \mu / \mathrm{ml}$ ). The coefficients of variation (CVs) for these assays were 1-2\% (TC, HDL, TG, FBG, CRP, $\left.\mathrm{fT}_{3}, \mathrm{fT}_{4}, \mathrm{TSH}\right), 2-3 \%$ (LDL), $2-4 \%\left(\mathrm{ApoA}_{1}\right.$ and $\mathrm{ApoB}$ ), 4-7\% (Lp(a), Tg-Ab, and TPO-Ab).

Brachial artery ultrasonography The vascular studies of the brachial artery were performed noninvasively, as we have previously described $(8,12)$. High resolution ultrasound was used to measure changes in arterial diameter in response to reactive hyperemia (with increased flow producing an endotheliumdependent stimulus to vasodilation; FMD) and to glyceryl trinitrate (GTN, an endothelium-independent vasodilator; GTN-induced dilation) (128XP/10 with a 7.0 MHZ linear array transducer: Acuson, Mountain View, CA, USA). The intra- and interobserver variability in our laboratory for repeated measurements of artery diameter are $0.09 \pm 0.10$ and $0.08 \pm 0.13 \mathrm{~mm}$ respectively. The CV for FMD measurements over time is $7.8-9.2 \%$; the power of the study to justify the selected sample size is $83.7 \%$, and the resolving power of the method tested on 'phantom arteries' is $8.6 \mu \mathrm{m}$.

The subjects rested in the supine position for $10 \mathrm{~min}$ before the first scan and remained supine throughout the study. The target artery (the brachial $2-10 \mathrm{~cm}$ above the elbow) was scanned in longitudinal section, and the center of the vessel was identified when the clearest images of anterior and posterior walls of the artery were obtained. The transmit zone was set to the level of the anterior vessel wall. Depth and gain settings were optimized to identify the lumen to vessel wall interface. Images were magnified with the resolution box function leading to a television line width of $\sim 0.05 \mathrm{~mm}$. Machine settings were kept constant during each study.

Flow increase was induced by inflation of a blood pressure tourniquet placed around the forearm distal to the target artery to $300 \mathrm{mmHg}$. The cuff was released after $5 \mathrm{~min}$ and, after cuff deflation, the artery was scanned continuously for $90 \mathrm{~s}, 15 \mathrm{~min}$ was allowed for vessel recovery, sublingual GTN (400- $\mu$ g spray) was then administered and, 5 min later, the last scan was done. The electrocardiogram was monitored continuously.

Vessel diameter was measured by two observers, unaware of clinical details and the stage of the experiment. The arterial diameter was measured at a fixed distance from an anatomical maker, such as a bifurcation, with ultrasonic calipers. Measurements were taken from the anterior to the posterior ' $m$ ' line at end diastole, incident with the $R$-wave on the electrocardiogram. The mean diameter was calculated from four cardiac cycles. For the hyperemia scan, vessel diameter was measured 45-60 s after cuff release. Diameter changes were derived as percent change relative to the first baseline scan $(100 \%)$. Baseline blood flow (measured during the first baseline scan) was estimated by multiplying angle-corrected, pulsed Doppler recordings of the flow velocity integral by $\pi$ and the square of the radius of the artery. Reactive hyperemia was calculated as the maximum flow recorded in the first $15 \mathrm{~s}$ after cuff deflation divided by the flow during the resting (baseline) scan.

Exercise intervention All subjects underwent a supervised orientation and thereafter performed exercise on their own. Initially, subjects walked 25-30 min/day, 3-4 days/week, at a relatively low intensity of exercise $(\sim 60 \%$ of their individually determined maximal heart rate obtained during the 
Table 1 Clinical and biochemical characteristics in control subjects and in patients with subclinical hypothyroidism (sHT) before and 6 months after exercise training intervention.

\begin{tabular}{|c|c|c|c|c|}
\hline \multirow[b]{2}{*}{ Variables } & \multicolumn{2}{|c|}{ Patients with sHT } & \multicolumn{2}{|c|}{ Control subjects } \\
\hline & Before exercise $(n=30)$ & After exercise $(n=28)$ & Before exercise $(n=27)$ & After exercise $(n=25)$ \\
\hline Age (years) & $53.0 \pm 8$ & $53.5 \pm 9$ & $52.0 \pm 7$ & $52.5 \pm 10$ \\
\hline $\mathrm{BMI}\left(\mathrm{kg} / \mathrm{m}^{2}\right)$ & $24.7 \pm 1.5$ & $24.0 \pm 1.3$ & $23.2 \pm 1.6$ & $22.8 \pm 2.7$ \\
\hline Fasting plasma glucose $(\mathrm{mg} / \mathrm{dl})$ & $86.2 \pm 1.1$ & $85.3 \pm 1.3$ & $85.1 \pm 1.5$ & $86.0 \pm 1.9$ \\
\hline Mean arterial pressure $(\mathrm{mmHg})$ & $102 \pm 5$ & $97 \pm 7$ & $99 \pm 8$ & $98 \pm 7$ \\
\hline Total cholesterol $(\mathrm{mmol} / \mathrm{l})$ & $5.33 \pm 0.62$ & $4.26 \pm 0.68^{*}$ & $4.42 \pm 0.49^{\star}$ & $4.29 \pm 0.52^{*}$ \\
\hline LDL (mmol/l) & $3.47 \pm 0.65$ & $2.55 \pm 0.65^{\dagger}$ & $2.38 \pm 0.46^{\dagger}$ & $2.22 \pm 0.44^{\dagger}$ \\
\hline $\mathrm{HDL}(\mathrm{mmol} / \mathrm{l})$ & $1.15 \pm 0.29$ & $1.28 \pm 0.34$ & $1.33 \pm 0.37$ & $1.30 \pm 0.35$ \\
\hline Triglycerides (mmol/l) & $2.87 \pm 1.07$ & $1.76 \pm 0.94^{\dagger}$ & $1.46 \pm 0.71^{\dagger}$ & $1.51 \pm 0.68^{\dagger}$ \\
\hline Apolipoprotein A1 (g/l) & $1.21 \pm 0.33$ & $1.20 \pm 0.41$ & $1.33 \pm 0.36$ & $1.28 \pm 0.40$ \\
\hline Apolipoprotein B $(\mathrm{g} / \mathrm{l})$ & $1.22 \pm 0.38$ & $1.19 \pm 0.32$ & $1.12 \pm 0.26$ & $1.09 \pm 0.32$ \\
\hline C-reactive protein (mg/l) & $3.22 \pm 0.13$ & $1.63 \pm 0.08^{\dagger}$ & $1.71 \pm 0.09^{\dagger}$ & $1.65 \pm 0.11^{\dagger}$ \\
\hline $\mathrm{VO}_{2} \max (\mathrm{ml} / \mathrm{kg}$ per min) & $25.6 \pm 2.8$ & $35.0 \pm 3.8^{\dagger, \neq}$ & $30.4 \pm 3.3^{*}$ & $39.7 \pm 3.1^{\dagger, \|}$ \\
\hline $\mathrm{fT}_{3}(\mathrm{pmol} / \mathrm{l})$ & $5.52 \pm 1.60$ & $6.34 \pm 1.58$ & $6.27 \pm 1.63$ & $6.08 \pm 1.46$ \\
\hline $\mathrm{fT}_{4}(\mathrm{pmol} / \mathrm{l})$ & $13.77 \pm 3.58$ & $14.12 \pm 3.12$ & $14.45 \pm 2.78$ & $15.14 \pm 3.12$ \\
\hline TSH (mU/I) & $7.62 \pm 1.46^{\S}$ & $7.59 \pm 1.52^{\S}$ & $2.95 \pm 1.42$ & $2.66 \pm 1.29$ \\
\hline TPO-Ab (U/ml) & $428.5 \pm 211.2^{\S}$ & $395.7 \pm 210.4^{\S}$ & $20.6 \pm 11.35$ & $24.3 \pm 13.1$ \\
\hline $\mathrm{Tg}-\mathrm{Ab}(\mathrm{U} / \mathrm{ml})$ & $297.8 \pm 123.7^{\S}$ & $266.3 \pm 105.6^{\S}$ & $17.4 \pm 9.2$ & $16.2 \pm 10.3$ \\
\hline
\end{tabular}

${ }^{\star} P<0.05,{ }^{\dagger} P<0.01$ compared with sHT before exercise training; ${ }^{\ddagger} P<0.05,{ }^{\S} P<0.01$ compared with control group before and after exercise; ${ }^{\|} P<0.05$ compared with before exercise.

measurement of maximal oxygen consumption). As their exercise tolerance improved, the intensity and duration of walking were increased to $40-45 \mathrm{~min} /$ day, 4-6 days/week, at an intensity of $70-75 \%$ of maximal heart rate $(30-50 \%$ subjects were advised to walk/jog or jog continuously to reach their target heart rate range). The patients were allowed to carry out an additional exercise training at home, but compliance to training session had to be $>60 \%$ for eligibility.

Maximal oxygen consumption Maximal oxygen consumption $\left(\mathrm{VO}_{2}\right.$ max) was assessed with online computer-assisted open-circuit spirometry during incremental treadmill exercise as previously described in detail (13). Heart rate (electrocardiograph) was also measured throughout the protocol.

\section{Statistical methods}

Data are reported as the mean \pm s.D. Data among different groups were compared with ANOVA. Linear regression analyses were used to assess the relation between the exercise training-induced changes in plasma lipids, glucose, CRP, and FMD. Association between the absolute changes of endotheliumdependent or -independent arterial dilation and the absolute changes of other variables, such as TC, TG, CRP, was assessed by multiple regression analysis. A $P$ value $<0.05$ was considered significant. Lp(a) concentrations were log transformed before analysis. All analyses were carried out by using the statistical package SPSS 11.5 (Chicago, IL, USA).

\section{Results}

The clinical characteristics and biochemical results of the control subjects and subjects with sHT before and 6 months after the exercise training were given in Table 1. At baseline, TC, TG, LDL, CRP, TSH, TPO-Ab, and TG-Ab concentrations were significantly higher in patients with sHT than those in controls $(P<0.05)$. In both groups, other parameters, i.e. mean arterial pressure, $\mathrm{fT}_{3}$, $\mathrm{fT}_{4}$, did not differ between the two groups

Table 2 The results of brachial artery studies in control subjects and in patients with subclinical hypothyroidism (sHT) before and 6 months after exercise training intervention.

\begin{tabular}{|c|c|c|c|c|}
\hline \multirow[b]{2}{*}{ Variables } & \multicolumn{2}{|c|}{ Patients with sHT } & \multicolumn{2}{|c|}{ Control subjects } \\
\hline & Before exercise $(n=30)$ & After exercise $(n=28)$ & Before exercise $(n=27)$ & After exercise $(n=25)$ \\
\hline Baseline vessel (mm) & $3.79 \pm 0.58$ & $3.80 \pm 0.55$ & $3.82 \pm 0.63$ & $3.79 \pm 0.54$ \\
\hline Baseline flow (ml/min) & $82.68 \pm 33.43$ & $84.32 \pm 35.21$ & $82.54 \pm 35.28$ & $83.15 \pm 34.72$ \\
\hline Reactive hyperemia (\%) & $438.6 \pm 132.4$ & $441.8 \pm 127.0$ & $439.1 \pm 116.5$ & $442.4 \pm 122.8$ \\
\hline Flow-mediated dilation (\%) & $3.87 \pm 0.69$ & $5.08 \pm 0.73^{*, \dagger}$ & $5.98 \pm 0.97^{\star}$ & $6.13 \pm 0.87^{\star}$ \\
\hline GNT-induced dilation (\%) & $20.62 \pm 1.38$ & $22.17 \pm 1.44$ & $20.92 \pm 1.50$ & $21.76 \pm 1.28$ \\
\hline
\end{tabular}

${ }^{\star} P<0.001$ compared with patients with $\mathrm{SHT}$ before exercise training; ${ }^{\dagger} P<0.001$ compared with control before and after training. 


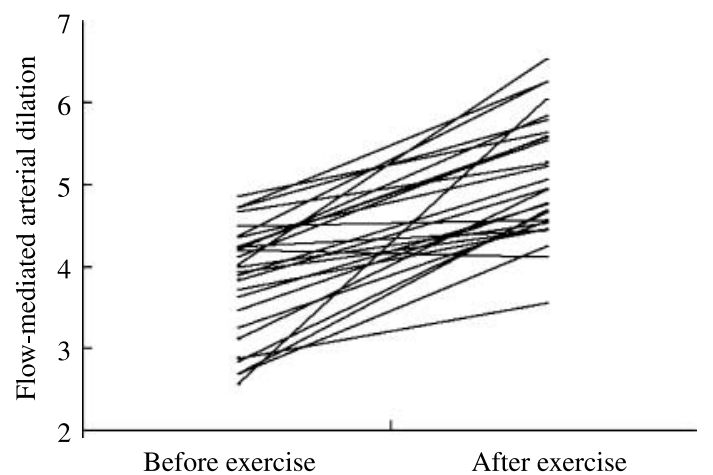

Figure 1 Changes of FMD before and after exercise training in 28 patients with sHT who completed the exercise training program.

$(P>0.05)$. Vascular characteristics of the groups were listed in Table 2. FMD among subjects with sHT was $3.87 \%$, which was significantly lower than that in controls $(5.98 \% ; P<0.001)$. The baseline vessel size (diameter), GNT-induced arterial dilation, and baseline flow were not significantly different between the two groups $(P>0.05)$.

After 6 months of exercise training, there was a remarkable increase in FMD (reaching 5.08\%, and $\Delta$ FMD $31.3 \%)$ and $\mathrm{VO}_{2}$ max $(36.7 \%)$ in subjects with sHT $(P<0.01)$. As shown in Fig. 1, most of the patients who completed the exercise training program showed a marked increase in FMD during the course of exercise intervention. Other vascular parameters such as baseline vessel and baseline flow, did not change markedly (Table 2). Some of metabolic control and other parameters improved with exercise training, and significant decreases in TC $(20 \%)$, LDL $(29 \%)$, TG (47.6\%), CRP (61.5\%) were observed over the exercise training period $(P<0.05)$. Other clinical parameters, such as thyroid function, TPO-Ab and Tg-Ab, did not significantly change during the exercise training period in patients with sHT (Table 1). In the control group, only $\mathrm{VO}_{2}$ max was found to increase significantly during the exercise training $(P<0.05)$, other variables, including FMD and GNT-induced dilation, failed to emerge as significant changes $(P>0.05)$.

To reveal possible causes of exercise training-induced FMD improvement, linear correlation coefficients were calculated between changes in metabolic and inflammatory parameters (TC, TG, LDL, CRP, $\mathrm{VO}_{2}$ max) and those of vascular reactivity (FMD and GNTinduced arterial dilation). The absolute changes in FMD showed significant correlation with the changes in LDL $(r=-0.596, P=0.001)$, TG $(r=-0.532$, $P=0.004) \quad$ and $\quad$ CRP $\quad(r=-0.511, \quad P=0.005$; Figs 2-4). No significant correlations were found between changes in FMD and those of $\mathrm{TC}$ and $\mathrm{VO}_{2}$ max in patients with sHT. There were no significant correlations between changes of FMD and changes of metabolic and inflammatory parameters in control subjects. Multiple regression analysis showed that changes of LDL, TG, and CRP were found to be significant determinants of changes of FMD in patients with sHT, and this association was not found in healthy women.

\section{Discussion}

Endothelial dysfunction is an important early event in atherogenesis (1). Both spatial and temporal correlations between endothelial dysfunction and coronary atherosclerosis have been shown in animal models and in humans (14-16). Impairment of endothelial function in early life could result in abnormal reactions between the vessel wall and platelets, neutrophils, and macrophages, and thus could contribute to the initial stages of atherogenesis (1). Impaired FMD has been observed in patients with coronary artery disease (1), type 2 diabetes mellitus (17), type 1 diabetes mellitus (18), and impaired fasting glucose (IFG) (8), hypertension (19), hypercholesterolemia (20), overt hypothyroidism, and sHT patients (5).

The present study showed that FMD using noninvasive measurement in sedentary patients with sHT decreased significantly at baseline, and multiple regression analysis showed that FMD was associated with TG, LDL, CRP, and TSH (data was not shown). This is in agreement with our previous results (5). After 6 months exercise training program, the impaired FMD in sedentary patients with sHT was significantly improved. This result is in good agreement with other training

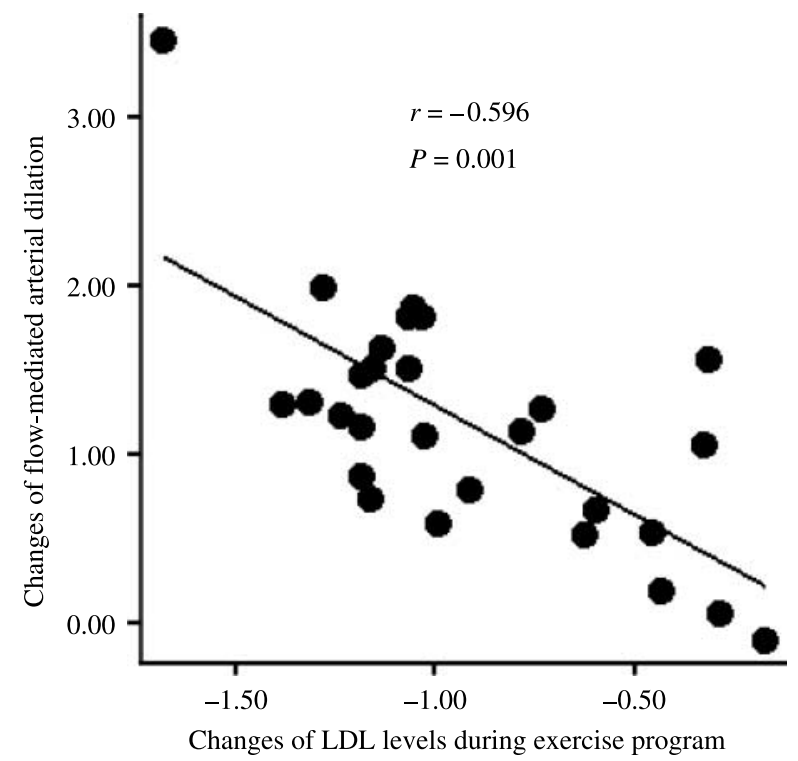

Figure 2 Linear regression analyses to evaluate correlation of change in FMD with change in LDL before and after exercise training program in 28 patients with $\mathrm{sHT}$ who completed the exercise training program. 


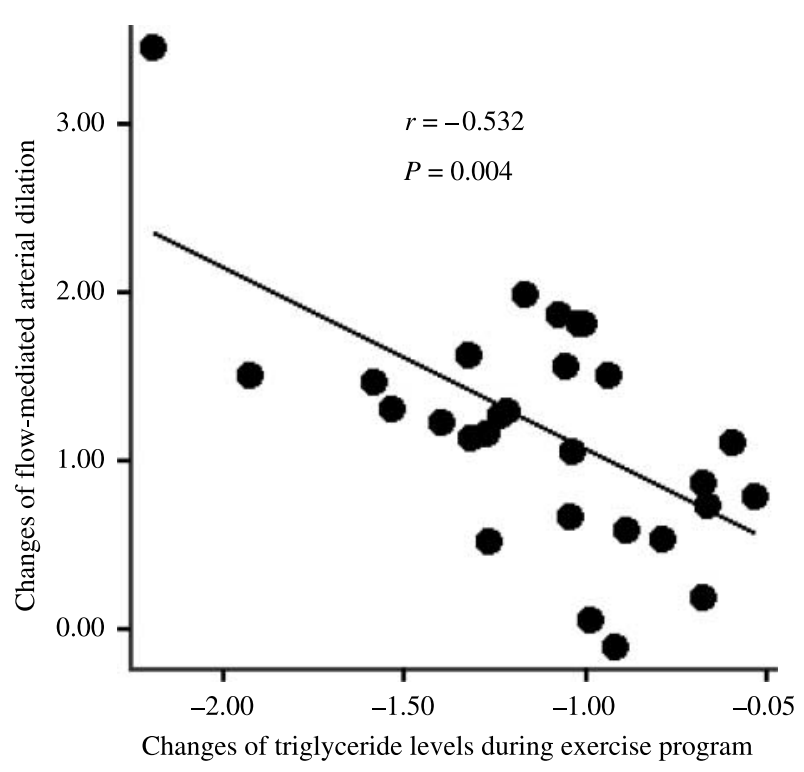

Figure 3 Linear regression analyses to evaluate correlation of change in FMD with change in triglyceride before and after exercise training program in 28 patients with $\mathrm{SHT}$ who completed the exercise training program.

studies in different patient cohorts (9-12). As far as we know, this is the first report in sHT patients' cohort.

There is no direct evidence of which mechanisms contribute to the functional improvement of the vasculature in patients with sHT in our study. Most authors have discussed the role of increased shear stress, which affects the vascular NO system in many ways $(1,21)$. Endothelial L-arginine uptake, the substrate of NO production, is increased (22). Further, NO synthase gene expression in endothelial cells is augmented (23). Training could have increased the antioxidative capacity by increased expression of the potent antioxidative extracellular enzyme superoxide dismutase, as shown in animal studies (24). This may partially contribute to the improvement of endothelial function during the exercise training period in patients with sHT.

Endothelial injury has been induced experimentally by hypercholesterolemia, particularly the LDL component (25). In clinical studies using invasive techniques, hypercholesterolemic adults have been shown to have impaired endothelium-dependent responses to a variety of pharmacologic stimuli, including infusions of acetylcholine $(26,27)$. In type 2 diabetes, FMD was negatively associated with LDL (12). Besides, FMD was inversely related to plasma TG in healthy individuals (28). In the present study, after 6 months of exercise training period, a significant decrease in LDL levels and TG was observed, and their changes are in a significant correlation with the change in FMD. These can also contribute to the improvement of endothelial function in patients with sHT.
CRP has been recently considered as a potential contributor to inflammatory diseases including atherosclerosis as well as a marker of cardiovascular risk (29). More recently, one study showed that low-grade systemic inflammation caused endothelial dysfunction in sHT patients with Hashimoto's thyroiditis (30). In the present study, our results showed that CRP decreased markedly, and the absolute change of CRP was negatively associated with change of FMD during the exercise training course in patients with sHT. Therefore, this may partially explain the improvement of endothelial function during exercise training course in patients with sHT.

In this study, we only found that exercise training can improve endothelial function in patients with sHT, but not in healthy subjects. The mechanisms are not clear completely. The possible explanations are as follows: i) the baseline FMD is significantly lower in patients with sHT than that in healthy women, ii) the endothelial function may be benefit from the improvement of lipid metabolism and inflammation during the exercise training period in sHT patients, iii) a longer period of training may be required to have a detectable improvement of endothelial function.

Consistent with several previous findings in prediabetes (8) and diabetes as well as nondiabetic patients (31), we did not find any association between $\mathrm{VO}_{2}$ max and endothelial function. The mechanism is not clear. This may be related to the narrow range of differences in $\mathrm{VO}_{2}$ max among individuals.

Some limitations of the present study should be mentioned. First, we did not measure the plasma insulin level, the relation between insulin or insulin resistance,

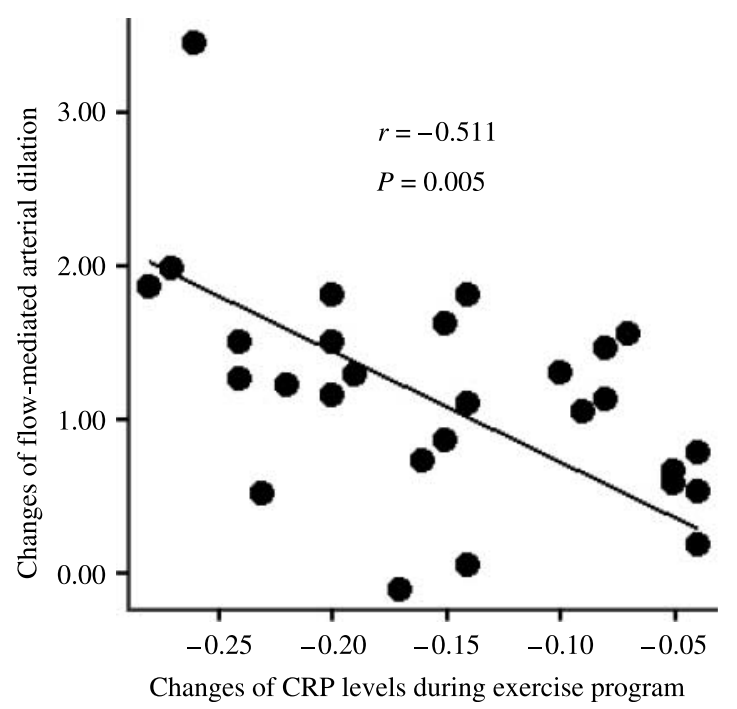

Figure 4 Linear regression analyses to evaluate correlation of change in FMD with change in CRP before and after exercise training program in 28 patients with $\mathrm{SHT}$ who completed the exercise training program. 
and endothelium-dependent arterial dilation before and after exercise training could not be evaluated. Insulin resistance is associated with endothelial dysfunction and cardiovascular risk (32). Secondly, the number of study subjects is relatively small. It is difficult to exclude completely the bias of the results, which should be confirmed in larger studies.

In summary, these results showed that regular aerobic exercise training can improve FMD in patients with sHT, although the mechanism for the improvement of FMD has not been explained completely.

\section{Declaration of interest}

There is no conflict of interest that could be perceived as prejudicing the impartiality of the research reported.

\section{Funding}

This research did not receive any specific grant from any funding agency in the public, commercial, or not-for-profit sector.

\section{References}

1 Boneti PO, Lerman LO \& Lerman A. Endothelial dysfunction, a marker of atherosclerotic risk. Arteriosclerosis, Thrombosis, and Vascular Biology 200323 168-175.

2 Rubanyi GM. The role of endothelium in cardiovascular homeostasis and diabetes. Journal of Cardiovascular Pharmacology 199322 S1-S14.

3 Kvetny J, Heldgaard PE, Bladbjerg RM \& Gram J. Subclinical hypothyroidism is associated with a low-grade inflammation, increased triglyceride levels and predicts cardiovascular disease in males below 50 years. Clinical Endocrinology $200461232-238$.

4 Imaizumi M, Akahoshi M, Ichimaru S, Nakashima E, Hida A, Soda M, Usa T, Ashizawa K, Yokoyama N, Maeda R, Nagataki S \& Eguchi K. Risk for ischemic heart disease and all-cause mortality in subclinical hypothyroidism. Journal of Clinical Endocrinology and Metabolism 200489 3365-3370.

5 Xiang GD, Sun HL, Cheng ZS \& Zhao LS. Changes in plasma concentration of osteoprotegerin before and after levothyroxine replacement therapy in hypothyroid patients. Journal of Clinical Endocrinology and Metabolism 200590 5765-5768.

6 Taddei S, Caraccio N, Virdis A, Dardano A, Versari D, Ghiadoni L, Salvtti A, Ferrannini E \& Monzani F. Impaired endotheliumdependent vasodilatation in subclinical hypothyroidism: beneficial effect of levothyroxine therapy. Journal of Clinical Endocrinology and Metabolism $2003 \mathbf{8 8} 3731-3737$.

7 Cooper DS. Clinical practice. Subclinical hypothyroidism. New England Journal of Medicine 2001345 260-265.

8 Xiang GD \& Wang YL. Regular aerobic exercise training improves endothelium-dependent artery dilation in patients with impaired fasting glucose. Diabetes Care 200427 801-802.

9 Fuchsjager-Mayerl G, Pleiner J, Wiesinger GF, Sieder AE, Quittan M, Nuhr MJ, Francesconi C, Seit H, Francesconi M, Schmetterer L \& Wolzt M. Exercise training improves vascular endothelial function in patients with type 1 diabetes. Diabetes Care 200225 1795-1780.

10 Maiorana A, O’Driscoll G, Cheetham C, Dembo L, Stanton K, Goodman C, Taylor K \& Green D. The effect of combined aerobic and resistance exercise training on vascular function in type 2 diabetes. Journal of the American College of Cardiology 200138 860-866.
11 Rinder MR, Spina RJ \& Ehsani AA. Enhanced endotheliumdependent vasodilation in older endurance-trained men. Journal of Applied Physiology 200088 761-766.

12 Xiang GD \& Wu YH. Apolipoprotein e4 allele and endotheliumdependent arterial dilation in type 2 diabetes mellitus without angiopathy. Diabetologia $2003 \mathbf{4 6} 514-519$.

13 Tanaka H, DeSouza CA, Jones PP, Stevenson ET, Davy KP \& Seals DR. Greater rate of decline in maximal aerobic capacity with age in physically active vs sedentary healthy women. Journal of Applied Physiology 199783 1947-1953.

14 Mclenacham JM, Vita JA, Fish RD, Treasure CB, Cox DA, Ganz P \& Selwyn AP. Early evidence of endothelial dysfunction at coronary branch points. Circulation 199082 1169-1173.

15 Harrison DG, Armstrong MI, Freman PC \& Heistad DD. Restoration of endothelium-dependent relaxation by dietary treatment of atherosclerosis. Journal of Clinical Investigation $1987 \mathbf{8 0}$ 1808-1811.

16 Fish RD, Nabel EG, Selwyn AP, Ludmer PL, Mudge GH, Kirshenbaum JM, Schoer FJ, Alexander RW \& Ganz P. Responses of coronary arteries of cardiac transplant patients to acetylcholine. Journal of Clinical Investigation 198881 21-31.

17 Xiang GD, Xu L, Zhao LS, Yue L \& Hou J. The relationship between plasma osteoprotegerin and endothelium-dependent arterial dilation in type 2 diabetes. Diabetes 200655 2126-2131.

18 Xiang GD, Sun HL \& Zhao LS. Changes of osteoprotegerin before and after insulin therapy in type 1 diabetic patients. Diabetes Research and Clinical Practice 200776 199-206.

19 Panza JA, Quyyumi AA, Brush JE \& Epstein SE. Abnormal endothelium-dependent vascular relaxation in patients with essential hypertension. New England Journal of Medicine 1990 $32322-27$.

20 Steinberg HO, Bayazeed OB, Hook G, Johnson A, Cronin J \& Baron AD. Endothelial dysfunction is associated with cholesterol levels in the high normal range. Circulation 199796 3287-3297.

21 Gielen S, Schuler G \& Hambrecht R. Exercise training in coronary artery disease and coronary vasomotion. Circulation 2001103 E1-E6.

22 Posch K, Schmidt K \& Graier WF. Selective stimulation of L-arginine uptake contributes to shear stress-induced formation of nitric oxide. Life Sciences 199964 663-670.

23 Yang AL, Tsai SJ, Jiang MJ, Jen CJ \& Chen HI. Chronic exercise increases both inducible and endothelial nitric oxide synthase gene expression in endothelial cells of rats aorta. Journal of Biomedical Science 20029 145-155.

24 Fukai T, Siegfried MR, Ushio-Fukai M, Cheng Y, Kojda G \& Harrison DG. Regulation of the vascular extracellular superoxide dismutase by nitric oxide and exercise training. Journal of Clinical Investigation 2000105 1631-1639.

25 Galle J, Bassenge E \& Busse R. Oxidized low-density lipoproteins potentiate vasoconstrictions to various agonists by direct interaction with vascular smooth muscle. Circulation Research 199066 1287-1293.

26 Drexler H, Zeiher AM, Meinzer K \& Just H. Correlation of endothelial dysfunction in coronary microcirculation of hypercholesterolemic patients by L-arginine. Lancet 1991338 1546-1550.

27 Vitu JA, Ireasure CB, Nabel IG, Melenaehan JM, Fish RD, Yeung AC, Vekyein VI, Selwyn AP \& Ganz P. Coronary vasomotor response to acetylcholine relates to risk factors for coronary artery disease. Circulation 198181 491-497.

28 Rasmussen JG, Eschen RB, Aardestrup IV, Dethlefsen C, Griffin BA \& Schmidt EB. Flow-mediated vasodilation: variation and interrelationships with plasma lipids and lipoproteins. Scandinavian Journal of Clinical and Laboratory Investigation 2008 20 1-5.

29 Ferri C, Croce G, Cofini V, De Berardinis G, Grassi D, Casale R, Properzi G \& Desideri G. C-reactive protein: interaction with the vascular endothelium and possible role in human atherosclerosis. Current Pharmaceutical Design 200713 1631-1645. 
30 Taddei S, Caraccio N, Virdis A, Dardano A, Versari D, Ghiadoni L, Ferrannini E, Salvetti A \& Monzani F. Low-grade systemic inflammation causes endothelial dysfunction in patients with Hashimoto's thyroiditis. Journal of Clinical Endocrinology and Metabolism 200691 5076-5082.

31 Miche E, Herrmann G, Nowak M, Wirtz U, Tietz M, Hürst M, Zoller B \& Radzewitz A. Effect of an exercise training program on endothelial dysfunction in diabetic and non-diabetic patients with severe chronic heart failure. Clinical Research in Cardiology 2006 $95 \mathrm{I} / 117-\mathrm{I} / 124$.

32 Hsueh WA \& Quinones MJ. Role of endothelial dysfunction in insulin resistance. American Journal of Cardiology 200392 10-71.

Received 5 August 2009

Accepted 27 August 2009 